\title{
Integrative PET: a new concept for outcome prediction in lymphoma
}

\author{
Michel Meignan ${ }^{1} \cdot$ Anne-Ségolène Cottereau
}

Received: 8 July 2015/Accepted: 13 July 2015/Published online: 4 August 2015

(C) Italian Association of Nuclear Medicine and Molecular Imaging 2015

Integrative FDG-PET in lymphoma is a new concept which can be defined as a method of prognostic imaging: PET imaging data are combined with data from other fields (clinical, biological, molecular) or other imaging techniques to get a better risk stratification of the patients. New treatments have drastically improved the outcomes in the most frequent lymphoma subtypes such as Hodgkin Lymphoma (HL) and Diffuse large B cell lymphoma (DLBCL) of follicular lymphoma (FL); however, a small percentage of patients is at high risk of treatment failure or relapses and cannot be selected accurately by the classic prognostic factors. Treatment of lymphoma is now a delicate balance between the risk of under treatment and over treatment. Therapy should be tailored to maintain or improve the cure rate in patient with high risk factors and to reduce the toxicity of drugs or radiations in low-risk patient.

New prognosticators are under investigation in lymphoma before treatment or after the first few cycles of chemotherapy (interim evaluation). Before treatment, molecular biomarkers characterizing the tumour genetic status can be obtained from the biopsied materials and have been evaluated as "a priori" prognostic factors. Similarly, baseline imaging could identify groups of treatment-resistant patients with a risk of poor outcome. By contrast, interim imaging early predicts treatment outcome and constitutes a chemosensitivity appraisal of the tumour in a specific patient. Both approaches are complementary.

Michel Meignan

michel.meignan@aphp.fr; michel.meignan@hmn.aphp.fr

1 LYSA Imaging, Nuclear Medicine Department, Hôpital Henri Mondor, Créteil, France

2 Nuclear Medicine Department, Centre Henri Becquerel, Rouen, France
In this regard, interim FDG-PET (iPET) has been proposed for early risk stratification in lymphoma and the first results published ten years ago were enthusiastic. However in advanced stage HL, more recent data have reported that the 5-year PFS of iPET is $23 \%$ in PET positive patients and less than $90 \%$ in PET negative when the Deauville criteria are used for PET reporting. The NPV (85-90 \%) and PPV (70-75\%) do not reach the values which were reported initially. The issue is further complex in DLBCL since in a large prospective series that has included more than 800 patients and used the $\triangle \mathrm{SUV}_{\max }$ for iPET reporting, the 2-year TTF was $47 \%$ in PET positive patients and $79 \%$ in the PET negative group.

More recently, quantitative baseline PET has been proposed as an alternative to iPET for outcome prediction in an effort to obtain prognostic information at staging without doing another PET scan. Quantitative PET requires a strict adherence to international guidelines for FDG-PET in oncology but the major clues are biological (incorrect timing of the acquisition) or due to human errors (versatile use of different acquisition or reconstruction parameters, incorrect reporting of injected activity and erroneous localization of the involved sites). Indeed, in many countries, the equipments are strictly controlled by manufacturers and health agencies. The prognostic value of different derived metrics (metabolic tumour volume, SUV $\max$ and Total Lesion Glycolysis, TLG) has been assessed in HL, DLBCL and recently in primary mediastinal B cell lymphoma (PMBCL) and peripheral $\mathrm{T}$ cell lymphoma (PTCL). Although MTV and TLG were identified as interesting prognostic factors, they do not allow a sufficient categorization of patients to change treatment only on this approach.

Integrative PET might overcome these difficulties. One of the simplest examples is combination of a quantitative parameter with a clinical index. In a retrospective study 
including 108 patients with PTCL, MTV was predictive of outcome. 2-year PFS was $26 \%$ in patients with large volume and $71 \%$ in patients with small volume. However, when this parameter was combined with the prognostic index for T cell lymphoma (PIT), the risk stratification was improved since one-third of patients with 2 adverse factors could be stratified in a high risk group with a $19 \% 2$-year PFS and one-third had an excellent outcome [1]. Baseline PET parameters can be combined with the response to treatment. In a small series of patients with Hodgkin lymphoma, MTV was predictive of outcome [2]. With a $225 \mathrm{~cm}^{3}$ cut-off, patients with a large MTV had a poor outcome with a $42 \%$ 4-year PFS versus $85 \%$ for the small volume. When combined with the response evaluated after 2 cycles of chemotherapy with interim PET using the $\Delta \mathrm{SUV}_{\text {max }}$ approach, the patients could be stratified in more discriminant risk categories: high-risk patients with high volume and poor interim response had a $20 \%$ 4-year PFS, whereas low-risk patients had a $92 \%$ 4-year PFS. This combination improves the prognostic value of interim PET and allows the identification of $60 \%$ of patients with a very low risk of treatment failure $(6 \%)$ and a very small group of patients with a particularly high risk $(80 \%$ of progression at 1 year).

In PMBCL, it has been shown recently in a 103 patients prospectively study that TLG was a strong predictor of outcome. However, the positive predictive value of the high TLG was only $36 \%$. Combining these data with end treatment response evaluated by PET with Deauville criteria splits the group of patients with high volume, allowing the identification of a subgroup with a poor response and a poor outcome. The PPV increased to $47 \%$.

Striking examples of integrative PET are coming from biological and molecular data. An extensive research is currently ongoing to investigate biological and molecular markers as prognostic factors in every subtype of lymphomas. Many studies are published but conflicting results explain that until now they are not used in routine. For instance, some authors have reported $\mathrm{Bcl} 2$ as a strong prognostic factor in DLBCL, whereas others found it was of little prognostic value. Reproducibility of manual or visual Immunohistochemistry (IHC) scoring and lack of agreement on the optimal positivity thresholds and expression throughout the laboratories have been identified as potential pitfalls. Moreover, in this group of heterogeneous diseases, the marker evaluation is for now limited to the biopsied material. Therefore, the prognostic values obtained is usually much lower than the prognostic values obtained with interim PET which scans all the sites of increase glucose metabolism and gives a global evaluation of the risk. Indeed, combination of biological and molecular data with PET data, i.e. two different methods of investigating the molecular characteristics of the cells, from gene expression profiling to metabolism, seems logical. In DLBCL, some studies have tried to define new predictive models combining PET data and gene expression profiling $(\mathrm{GCB} / \mathrm{ABC})$ or expression of relevant biomarkers in tumour cells $(\mathrm{BCl} 2)$ [3]. Combining the GCB and ABC subtypes with the results of interim PET, it has been possible to identify two risk groups: a good risk group (patients with a good response and GCB subtype) and a bad risk group (all ABC patients and slow-responder GCB patients). A multivariate analysis demonstrated that the GCB/ ABC subtype, metabolic response on iPET and age-adjusted IPI were three independent factors that accurately predicted both PFS and OS in patients treated with rituximab chemotherapy. The same approach was recently investigated by Agostinelli in a large group of 310 patients with advanced stage HL treated with ABVD. It has been reported that the presence of different markers in the accessory cells (CD68KP-1 and PD1) and in RS cells (STAT-1) splits iPETnegative patients in two groups: iPET-negative with a low molecular risk profile and a good outcome $(94 \%$ 2-year PFS) and iPET-negative patients with a high molecular risk profile and a poor outcome (23\% 2-year PFS). This is an important issue since we know that $10-15 \%$ of PET negative patients have a poor outcome and this could be a mean for an early identification of this subgroup.

FDG-PET can be now combined with diffusion-weighted magnetic resonance imaging resulting in new imaging parameters that will probably help patient outcome prediction when used in this integrated process.

In conclusion, integrative PET is a holistic approach aimed to obtain new prognostic parameters. From the first results which have been published, we can expect that it will lead to a better risk stratification of the patients allowing tailoring therapeutic strategy.

\section{Compliance with ethics standards}

Conflict of interest The authors declare that they have no conflicts of interest to disclose.

Ethical standards This article does not contain any studies with human or animal subjects performed by any of the authors.

\section{References}

1. Kanoun S, Rossi C, Berriolo-Riedinger A et al (2014) Baseline metabolic tumour volume is an independent prognostic factor in Hodgkin lymphoma. Eur J Nucl Med Mol Imaging 41:1735-1743

2. Cottereau AS, Becker S, Broussais F et al (2015) Pronostic value of Baseline Metabolic Tumor Volume (MTV0) measured on FDG PET/CT in patients with peripheral T-cell Lymphoma (PTCL) 13ICML Oral Presentations. Hematol Oncol 33:100-180. doi:10. 1002/hon. 2227

3. Lanic H, Mareschal S, Mechken F et al (2012) Interim positron emission tomography scan associated with international prognostic index and germinal center B cell-like signature as prognostic index in diffuse large B-cell lymphoma. Leuk Lymphoma 53:34-42 